\title{
Spontaneously Improving Occipitocondylar Hyperplasia: A Case Report
}

\author{
Laszlo Nagy ${ }^{1}$ Coby Ray ${ }^{2}$ \\ ${ }^{1}$ Department of Pediatric Neurosurgery, Texas Tech University Health \\ Sciences Center, Lubbock, Texas, United States \\ ${ }^{2}$ School of Medicine, Texas Tech University Health Sciences Center, \\ Lubbock, Texas, United States \\ J Neurol Surg Rep 2014;75:e141-e143.
}

Address for correspondence Coby Ray, MBA, 4102 24th St Suite 410, Lubbock, TX 79410, United States (e-mail: coby.ray@ttuhsc.edu).

\begin{abstract}
This case report presents a pediatric patient with the extremely rare craniovertebral junction anomaly of occipitocondylar hyperplasia. This 4-year old boy presented with macrocephaly and mild to moderate pontomedullary compression from the unilateral occipitocondylar hyperplasia. Based on the asymptomatic clinical presentation, it was

Keywords

- hyperplasia

- occipital condyle

- occipitocondylar

- skull base decided to follow the patient with serial magnetic resonance images without surgical intervention. Upon further imaging the occipitocondylar hyperplasia spontaneously resolved. This case report offers watchful waiting as an alternative treatment approach to surgical intervention as was reported in the literature previously. Possible pathophysiologic mechanisms are also briefly explored.
\end{abstract}

\section{Introduction}

Since the introduction of computed tomography (CT) and magnetic resonance imaging (MRI) for the care of neurosurgical patients, a range of craniovertebral anomalies have been reported. ${ }^{1}$ An extremely rare craniovertebral junction anomaly is the presence of occipitocondylar hyperplasia, which has only been reported once before in the literature. ${ }^{2}$

\section{Case Presentation}

\section{History}

The patient is a white full-term boy with no known prenatal concerns. At birth he was found to have macrocephaly but was otherwise healthy. The initial diagnosis of idiopathic macrocephaly was reported by the family. In the first few years of life he was reported to be developmentally delayed in both movement and speech. He had no signs of a genetic syndrome or disease. The patient presented to the neurosurgery office at age 3 for follow-up for macrocephaly. The fact that the patient's family resides in a fairly closed religious community, which seems to have been very tolerant of the developmental delay and the macrocephaly, as well as the lack of other neurologic deficits, contributed to the lack of care prior to his presentation at our facility. The family reported that the developmental delay was improving continuously as the macrocephaly also resolved. At presentation his physical examination was unremarkable except for the increased head circumference of $54.50 \mathrm{~cm}$. To further evaluate the macrocephaly, an MRI of the head and an X-ray of the cervical spine were ordered. This led to a follow-up CT scan of the craniocervical junction.

\section{Radiographic Findings}

The flexion and extension X-ray were unremarkable. The CT scan ( - Fig. 1) and MRI ( - Fig. 2) demonstrated stenosis at the foramen magnum with a hypertrophic left occipitocondylar joint. This was protruding into the foramen magnum, thus compressing and distorting the pontine and medullary brainstem. He also had subtle ventriculomegaly and enlarged subarachnoid spaces. Despite these findings, no obstructive hydrocephalus was noted. Fluid pathways were seemingly patent, which correlated clinically with the patient's lack of neurologic findings suggestive of increased intracranial pressure. received

January 23, 2014 accepted after revision April 3, 2014 published online May 28, 2014
DOI http://dx.doi.org/ 10.1055/s-0034-1376426. ISSN 2193-6358. 〔c 2014 Georg Thieme Verlag KG
Stuttgart · New York

License terms

(1) $\Theta \circledast$ 


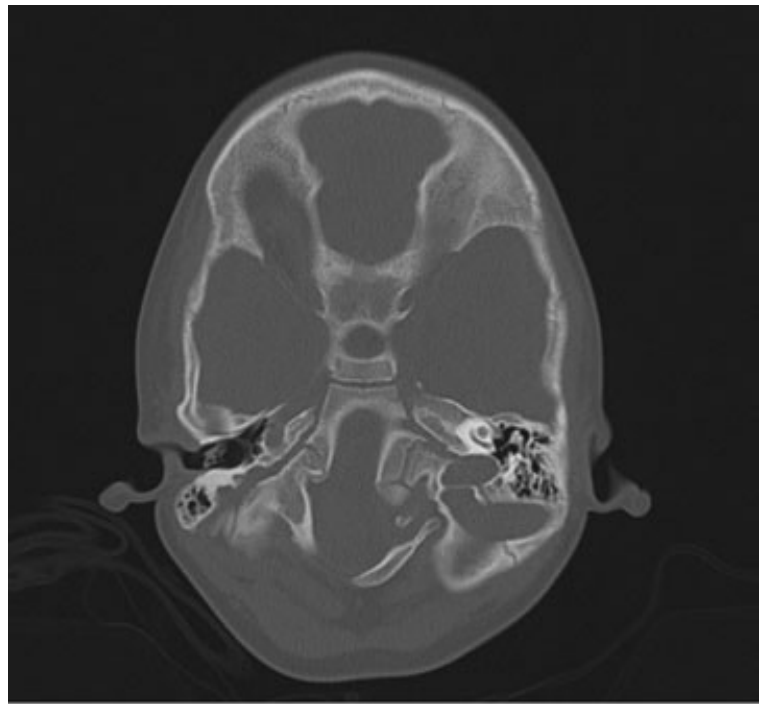

Fig. 1 Computed tomography scan demonstrating unilateral hyperplasia of the occipitocondylar.

\section{Examination}

Upon physical examination no associated symptoms were appreciated, specifically no headache, no swallowing difficulties, no cranial nerve abnormalities, and no ataxia. The cervical spine flexion-extension physical examination and X-ray were both negative. There were no signs of increased intracranial pressure, such as papilledema, on funduscopic examination.

\section{Follow-up Radiographic Findings and Examination}

At the 1-year follow-up, the 4-year-old still remained asymptomatic. A CT scan of the head (-Fig. 3) with three-dimen-

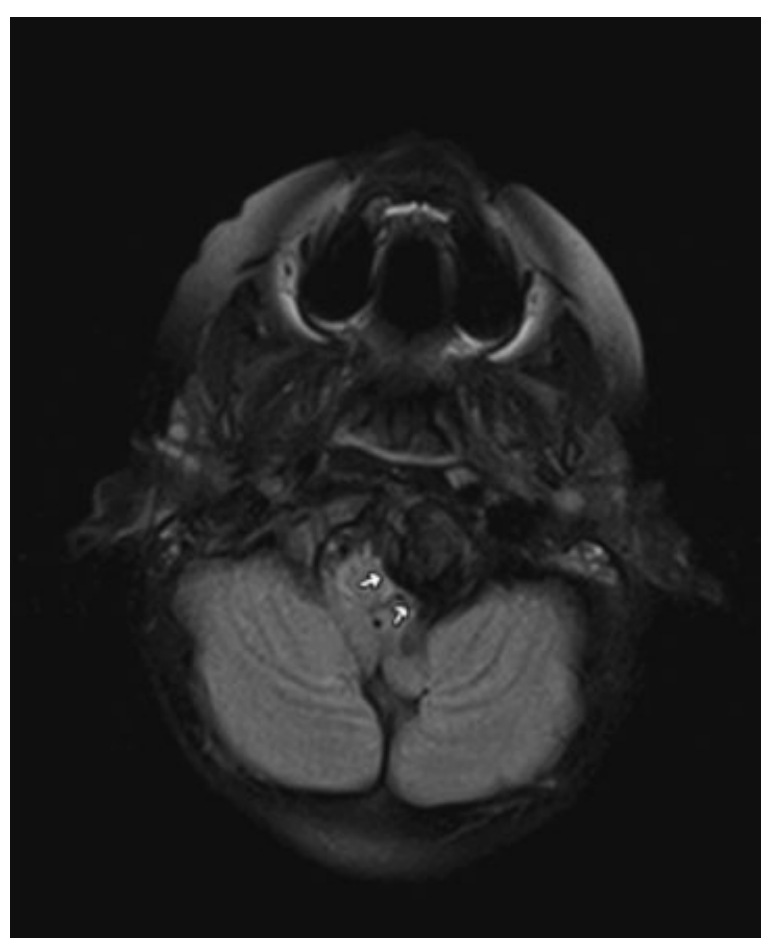

Fig. 2 Magnetic resonance imaging demonstrating unilateral hyperplasia of the occipitocondylar.

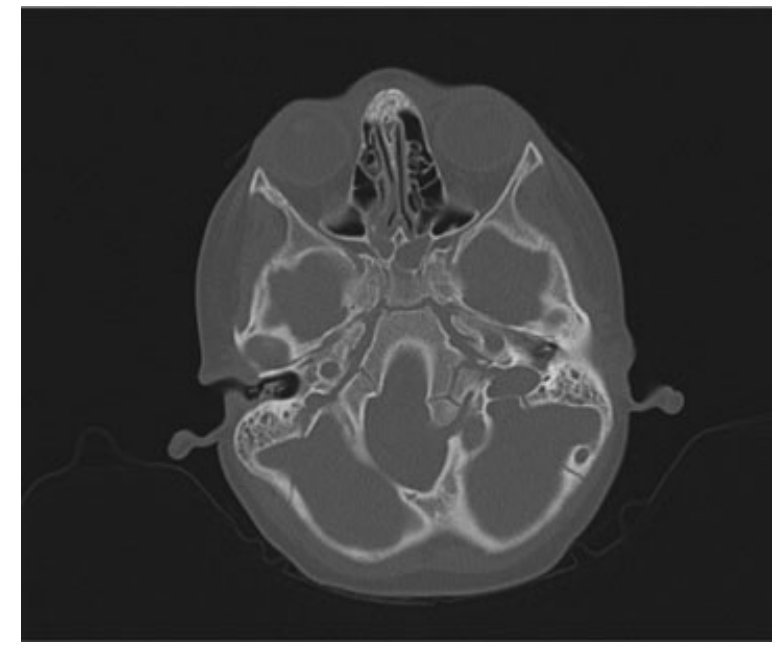

Fig. 3 Computed tomography scan demonstrating spontaneous resolution of the previously demonstrated unilateral hyperplasia of the occipitocondylar.

sional reconstruction demonstrated the occipitocondylar hyperplasia was slightly improved.

At the 3-year follow-up, an MRI of the head (-Fig. 4) demonstrated significant resolution of the occipitocondylar hyperplasia. Based on this finding and extensive discussions with the parents, it was decided to continue following him with yearly MRIs.

\section{Discussion}

A thorough understanding of the embryological development of the craniovertebral axis is essential in beginning to develop a full understanding of this disease process. At the initial developmental stage, the 01-03 vertebral primordia are separated from the $\mathrm{C} 1$ precursor by the proatlas. During development of the occipital bone, the two occipital condyles

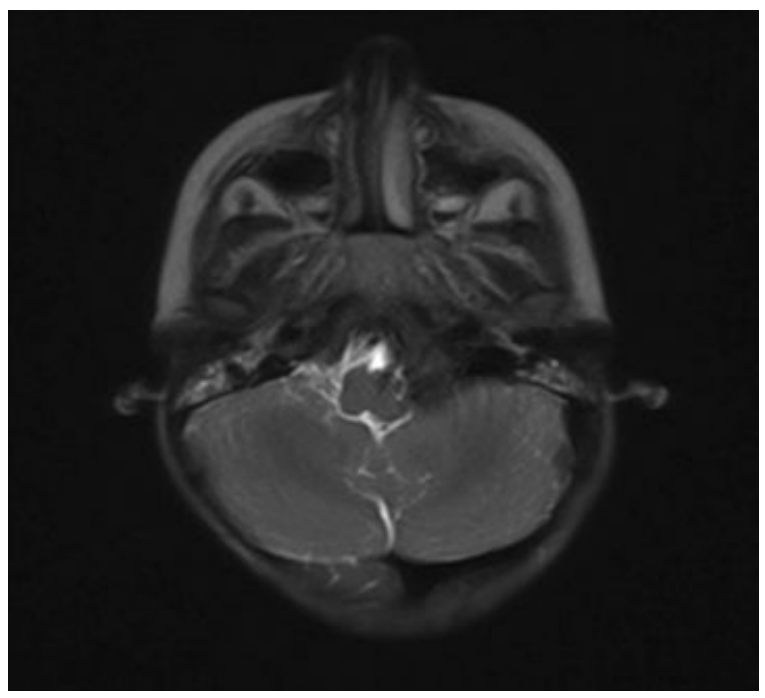

Fig. 4 Magnetic resonance imaging demonstrating spontaneous resolution of the previously demonstrated unilateral hyperplasia of the occipitocondylar. 
form when the neural arch of the proatlas divides into a rostral and caudal subdivision. The rostral portion of these vertebral primordia develops into the occipital condyle. The caudal segment becomes the tip of the dens and top of $\mathrm{C} 1 .^{3}$

The formation of the posterior fossa occurs as a result of the process of endocranial resorption, sutural growth, and bony accretion. ${ }^{4}$ The most important of these is the resorption. Synchondrosis growth also plays a significant role, even through the second decade of life. ${ }^{3,5}$ This continued occipital development and specifically the endocranial resorption is likely a major element of the spontaneous resolution of this patient's hyperplasia. Children who have craniobasal instrumentation and/or surgical history require close follow-up due to this continually evolving anatomy. In the same way, close follow-up is equally important for the conservative approach to management.

The fact that the patient did not continue to have the lesion despite normal anatomical growth of the craniocervical junction likely resulted from the created space for elongation and lateral retraction of the lesion. The physical relation of the pathologic and normally developing skull base resulted in elongation and lateral retraction of the pathologically affected area. This leads these authors to suggest the possible presence of a transient cause such as disproportionate local blood supply, focal metabolism, in utero positioning, or an improving unknown cause, which has abolished the pathologic configuration ending in an arrested lesion and the normal development eventually compensating for this lesion. However, these remain only hypotheses. Unfortunately, due to the rarity of these lesions, there is no research to date into the pathophysiology of this particular malformation.

One unique aspect of this case is found in the unilateral hyperplasia versus the previously reported bilateral presentation, ${ }^{2}$ as well as the asymptomatic presentation. The lack of physical examination findings, as well of the lack of any neurologic deficits, supports the use of the term asymptomatic in this patient. Although some variability from normal in the MRI scan is present, the patient did not report or demonstrate any associated signs or symptoms to warrant intervention. The developmental delay was consistently improving, and likely much of this delay could be attributed to the environment of his community and the lack of social interaction that resulted from his macrocephaly. The macrocephaly also is not a symptom of the occipital hyperplasia, but rather comorbidity. In the same way, the developmental delay was likely from the social isolation and unusual social experiences experienced by the patient due to his unusual appearance from the macrocephaly. This lack of signs or symptoms was a major component in deciding to treat conservatively with close observation without surgical intervention. At presentation and upon retrospective review, the decision to follow conservatively appears to have been the correct treatment option.

We recommend close follow-up of asymptomatic patients with radiographically demonstrated occipitocondylar hyperplasia. It is suggested that this observation regimen should consist of semiannual MRIs until the beginning of the second decade of life. This can then be altered to yearly MRIs until the lesion fully resolves or until the lesion demonstrates significant improvement.

This case report represents the second reported case of occipitocondylar hyperplasia and the first unilateral presentation. It also adds a significant component to the understanding of the natural history of the disease and brings to light the potential treatment option of following conservatively without surgical intervention.

\section{Disclosure}

The authors report no conflict of interest concerning the materials or methods used in this study or the findings specified in this article.

\section{References}

1 Menezes AH. Craniovertebral junction abnormalities. In: Albright LA, Pollack IF, Adelsen PD, eds. Principles and Practice of Pediatric Neurosurgery. New York, NY: Thieme Medical Publishers; 2008:395-414

2 Ohaegbulam C, Woodard EJ, Proctor M. Occipitocondylar hyperplasia: an unusual craniovertebral junction anomaly causing myelopathy. Case report. J Neurosurg 2005;103(4, Suppl):379-381

3 Menezes AH. Embryology, development, and classification of disorders of the craniovertebral junction. In: Bambakidis NC, Dickman CA, Spetzler RF, Sonntag Volker KH, eds. Surgery of the Craniovertebral Junction. New York, NY: Thieme Medical Publishers; 2013

4 Björk A. Cranial base development: a follow-up x-ray study of the individual variation in growth occurring between the ages of 12 and 20 years and its relation to brain case and face development. Am J Orthodontics 1955;41(3):198-225

5 Menezes AH. Evaluation and treatment of congenital and developmental anomalies of the cervical spine. Invited submission from the Joint Section Meeting on Disorders of the Spine and Peripheral Nerves, March 2004. J Neurosurg Spine 2004;1(2):188-197 